Supporting Information for

\title{
Structural Engineering of Graphitic Carbon Nitrides for Enhanced Metal-free PET-RAFT Polymerizations in Heterogeneous and Homogeneous Systems
}

\author{
Lei Zhang ${ }^{\mathrm{a}}$, Gang $\mathrm{Ye}^{\mathrm{a}, \mathrm{b},{ }^{*}}$, Xiaomei Huo ${ }^{\mathrm{a}}$, Shengming $\mathrm{Xu}^{\mathrm{a}, \mathrm{b}}$, Jing Chen ${ }^{\mathrm{a}, \mathrm{b}}$, Krzysztof Matyjaszewski ${ }^{\mathrm{c},{ }^{*}}$ \\ ${ }^{a}$ Collaborative Innovation Center of Advanced Nuclear Energy Technology, Institute of Nuclear and New Energy \\ Technology, Tsinghua University, Beijing, 100084, China \\ ${ }^{b}$ Beijing Key Lab of Radioactive Waste Treatment, Tsinghua University, Beijing 100084, China \\ ${ }^{c}$ Department of Chemistry, Carnegie Mellon University, 4400 Fifth Avenue, Pittsburgh \\ Corresponding Authors, E-mail: yegang@mail.tsinghua.edu.cn; matyjaszewski@cmu.edu
}




\section{Supporting Figures}

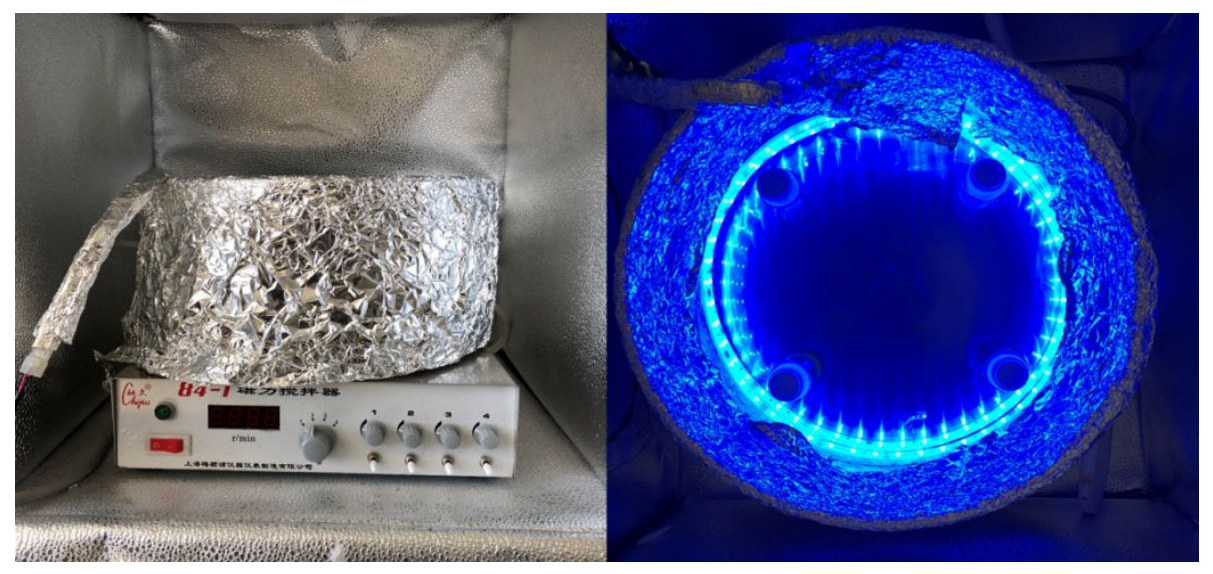

Figure S1. Photographs of the setup for PET-RAFT polymerization 

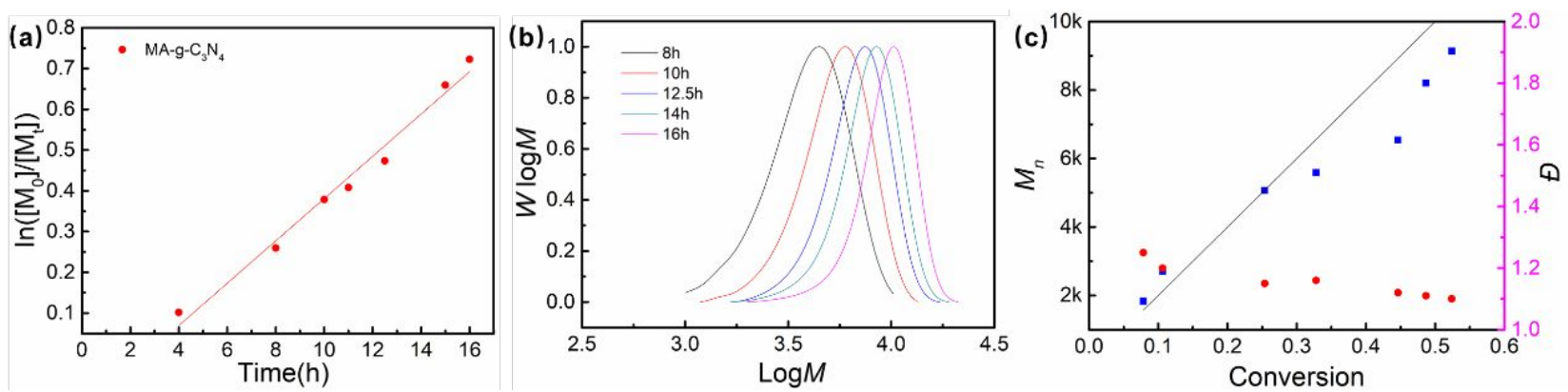

Figure S2. Kinetic study of MA-g- $\mathrm{C}_{3} \mathrm{~N}_{4}$ catalyzed PET-RAFT polymerization of MMA using CPADB as the chain transfer agent under blue LED irradiation $\left(\lambda_{\max }=465 \mathrm{~nm}, 3 \mathrm{~mW} / \mathrm{cm}^{2}\right)$. The molar ratio of $[\mathrm{MMA}] /[\mathrm{CPADB}]=200$ : 1 in $1 \mathrm{~mL}$ DMSO: (a) $\ln \left([\mathrm{M}]_{0} /[\mathrm{M}]_{\mathrm{t}}\right)$ versus irradiation time and the fitted kinetic curve; (b) GPC profiles of PMMA synthesized in the kinetic study; (c) Number average molecular weight $\left(M_{\mathrm{n}}\right)$ and dispersity $(\bigoplus)$ of PMMA obtained in the kinetic study. 


\section{Supporting Tables}

Table S1. Kinetic study on PET-RAFT polymerization of MMA catalyzed by TCA-g- $\mathrm{C}_{3} \mathrm{~N}_{4}$ under 3 $\mathrm{mW} / \mathrm{cm}^{2}$ blue LED irradiation.

\begin{tabular}{lllllllll}
\hline Entry & $\mathrm{g}_{-} \mathrm{C}_{3} \mathrm{~N}_{4}$ & {$[\mathrm{MMA}]:[\mathrm{CPADB}]$} & $\mathrm{m}_{\mathrm{g}-\mathrm{C} 3 \mathrm{~N} 4}$ & Time $(\mathrm{h})$ & $\alpha(\%)$ & $M_{\mathrm{n}, \text { theo }}$ & $M_{\mathrm{n}, \mathrm{GPC}}$ & $Ð$ \\
\hline 1 & TCA & $200: 1$ & 5 & 4 & 15 & 3,000 & 2,200 & 1.35 \\
2 & TCA & $200: 1$ & 5 & 6 & 20 & 4,100 & 4,000 & 1.21 \\
3 & TCA & $200: 1$ & 5 & 8 & 23 & 4,700 & 5,400 & 1.17 \\
4 & TCA & $200: 1$ & 5 & 10 & 32 & 6,400 & 6,900 & 1.13 \\
5 & TCA & $200: 1$ & 5 & 11 & 36 & 7,200 & 7,500 & 1.12 \\
6 & TCA & $200: 1$ & 5 & 12.5 & 43 & 8,600 & 8,400 & 1.11 \\
7 & TCA & $200: 1$ & 5 & 14 & 49 & 9,900 & 9,300 & 1.11 \\
8 & TCA & $200: 1$ & 5 & 15 & 55 & 11,000 & 10,100 & 1.10 \\
9 & TCA & $200: 1$ & 5 & 16 & 60 & 12,000 & 10,800 & 1.10 \\
\hline
\end{tabular}


Table S2. Kinetic study on PET-RAFT polymerization of MMA catalyzed by $\mathrm{MA}-\mathrm{g}-\mathrm{C}_{3} \mathrm{~N}_{4}$ under 3 $\mathrm{mW} / \mathrm{cm}^{2}$ blue LED irradiation.

\begin{tabular}{lllllllll}
\hline Entry & $\mathrm{g}_{-} \mathrm{C}_{3} \mathrm{~N}_{4}$ & {$[\mathrm{MMA}]:[\mathrm{CPADB}]$} & $\mathrm{m}_{\mathrm{g}-\mathrm{C} 3 \mathrm{~N} 4}$ & Time $(\mathrm{h})$ & $\alpha(\%)$ & $M_{\mathrm{n}, \text { theo }}$ & $M_{\mathrm{n}, \mathrm{GPC}}$ & $\oplus$ \\
\hline 1 & $\mathrm{MA}$ & $200: 1$ & 5 & 6 & 12 & 2,400 & 2,700 & 1.20 \\
2 & $\mathrm{MA}$ & $200: 1$ & 5 & 8 & 22 & 4,600 & 3,600 & 1.21 \\
3 & $\mathrm{MA}$ & $200: 1$ & 5 & 10 & 31 & 6,300 & 5,000 & 1.15 \\
4 & $\mathrm{MA}$ & $200: 1$ & 5 & 11 & 33 & 6,700 & 5,600 & 1.16 \\
5 & MA & $200: 1$ & 5 & 12.5 & 37 & 7,500 & 6,500 & 1.12 \\
6 & MA & $200: 1$ & 5 & 14 & 49 & 9,800 & 7,500 & 1.14 \\
7 & MA & $200: 1$ & 5 & 16 & 51 & 10,000 & 9,100 & 1.10 \\
\hline
\end{tabular}

